\title{
On a discussion of Volterra-Fredholm integral equation with discontinuous kernel
}

\author{
M. A. Abdou' , A. A. Soliman² and M. A. Abdel-Aty2 ${ }^{*}$
}

*Correspondence:

mohammed.abdallah@fsc.bu.edu.eg

${ }^{2}$ Department of Mathematics,

Faculty of Science, Benha University,

Benha, 13518, Egypt

Full list of author information is

available at the end of the article

\begin{abstract}
The purpose of this paper is to establish the general solution of a Volterra-Fredholm integral equation with discontinuous kernel in a Banach space. Banach's fixed point theorem is used to prove the existence and uniqueness of the solution. By using separation of variables method, the problem is reduced to Volterra integral equations of the second kind with continuous kernel. Normality and continuity of the integral operator are also discussed.
\end{abstract}

Keywords: Banach space, Volterra-Fredholm integral equation, Separation of variables method

AMS Subject Classification: Primary; 45L05; secondary; 46B45; 65R20

\section{Introduction}

It is well known that the integral equations govern many mathematical models of various phenomena in physics, economy, biology, engineering, and even in mathematics and other fields of science. The illustrative examples of such models can be found in the literature (see, e.g., [1-8]). Many problems of mathematical physics, applied mathematics, and engineering are reduced to Volterra-Fredholm integral equations, see [9-12].

Analytical solutions of integral equations either do not exist or are hard to compute $[13,14]$. Eventually, an exact solution is computable, the required calculations may be tedious, or the resulting solution may be difficult to interpret. Due to this, it is required to obtain an efficient numerical solution [15]. There are numerous studies in literature concerning the numerical solution of integral equations such as [16-22].

The basic motivation of this paper is to present a new class of integral equations arising from mathematical physics; the existence and uniqueness solution of Eq. (1) are discussed and proved in the space $L_{2}(\Omega) \times C[0, T], 0 \leq T<1$. Moreover, the normality and continuity of the integral operator are obtained. A numerical method is used to translate the Volterra-Fredholm integral Eq. (1) to Volterra integral equations of the second kind with the continuous kernel, the ideas are interesting, and this area caught the attention of many researchers, having so many applications.

The outline of the paper is as follows: The "Introduction" section is the introduction. In "The existence of a unique solution of the Volterra-Fredholm integral equation" section, the existence of a unique solution of the Volterra-Fredholm integral equation is discussed and proved using Picard's method and Banach's fixed point method. The

(c) The Author(s). 2020 Open Access This article is distributed under the terms of the Creative Commons Attribution 4.0 International License (http://creativecommons.org/licenses/by/4.0/), which permits unrestricted use, distribution, and reproduction in any medium, provided you give appropriate credit to the original author(s) and the source, provide a link to the Creative Commons license, and indicate if changes were made. 
"Separation of variables method" section includes the general solution of the VolterraFredholm integral equation by applying the method of separation of variables. A brief conclusion is presented in the "Conclusion and remarks" section.

Consider the following linear Volterra-Fredholm integral equation:

$$
\begin{gathered}
\mu \psi(x, t)-\lambda \int_{0}^{t} \int_{\Omega} \Phi(t, \tau) k(|x-y|) \psi(y, \tau) \mathrm{d} y \mathrm{~d} \tau=g(x, t), \\
\left(x=\bar{x}\left(x_{1}, x_{1}, \ldots, x_{n}\right), \quad y=\bar{y}\left(y_{1}, y_{1}, \ldots, y_{n}\right)\right),
\end{gathered}
$$

where $\mu$ is a constant which defined the kind of integral equation, and $\lambda$ is the constant which may be complex and has many physical meanings. The function $\psi(x, t)$ is unknown in the Banach space $L_{2}(\Omega) \times C[0, T], 0 \leq T<1$ [23-25], where $\Omega$ is the domain of integration with respect to position and the time $t \in[0, T]$ and is called the potential function of the Volterra-Fredholm integral equation. The kernel of time $\Phi(t, \tau)$ is continuous in $C[0, T]$, and the known function $g(x, t)$ is continuous in the space $L_{2}(\Omega) \times C[0, T], 0 \leq t \leq T$. In addition, the kernel of position $k(|x-y|)$ is a discontinuous function.

\section{The existence of a unique solution of the Volterra-Fredholm integral equation}

In this paper, for discussing the existence and uniqueness of the solution of Eq. (1), we assume the following conditions:

(i) The kernel of position $k(|x-y|) \in L_{2}(\Omega), x, y \in[\Omega]$ satisfies the discontinuity condition:

$$
\left\{\int_{\Omega} \int_{\Omega} k^{2}(|x-y|) \mathrm{d} x \mathrm{~d} y\right\}^{\frac{1}{2}}=k^{*}, \quad k^{*} \text { is a constant. }
$$

(ii) The kernel of time $\Phi(t, \tau) \in C[0, T]$ satisfies $|\Phi(t, \tau)| \leq M$, such that $M$ is a constant, $\forall t, \tau \in[0, T]$.

(iii) The given function $g(x, t)$ with its partial derivatives with respect to the position and time is continuous in the space $L_{2}(\Omega) \times C[0, T], 0 \leq \tau \leq T<1$, and its norm is defined by:

$$
\|g(x, t)\|=\max _{0 \leq t \leq T} \int_{0}^{t}\left(\int_{\Omega} g^{2}(x, \tau) \mathrm{d} x\right)^{\frac{1}{2}} \mathrm{~d} \tau=N, \quad N \text { is a constant. }
$$

Theorem 1 If the conditions (i)-(iii) are satisfied, then Eq. (1) has a unique solution $\psi(x, t)$ in the Banach space $L_{2}(\Omega) \times C[0, T], 0 \leq T<1$, under the condition:

$$
|\lambda|<\frac{|\mu|}{M k^{*}} .
$$

Proof To prove the existence of a unique solution of Eq. (1), we use the successive approximations method (Picard's method), or we can used Banach's fixed point theorem.

\section{Picard's method}

We assume the solution of Eq. (1) takes the form:

$$
\psi(x, t)=\lim _{n \rightarrow \infty} \psi_{n}(x, t),
$$


where

$$
\psi_{n}(x, t)=\sum_{i=0}^{n} H_{i}(x, t) ; \quad t \in[0, T] ; \quad n=1,2, \ldots
$$

where the functions $H_{i}(x, t), i=0,1, \ldots, n$ are continuous functions of the form:

$$
\left.\begin{array}{l}
H_{n}(x, t)=\psi_{n}(x, t)-\psi_{n-1}(x, t) \\
H_{0}(x, t)=g(x, t)
\end{array}\right\} .
$$

Now, we should prove the following lemmas:

Lemma 1 Under the conditions (i)-(iii), the series $\sum_{i=0}^{n} H_{i}(x, t)$ is uniformly convergent to a continuous solution function $\psi(x, t)$.

Proof We construct the sequences:

$$
\mu \psi_{n}(x, t)=g(x, t)+\lambda \int_{0}^{t} \int_{\Omega} \Phi(t, \tau) k(|x-y|) \psi_{n-1}(y, \tau) \mathrm{d} y \mathrm{~d} \tau ; \quad \psi_{0}(x, t)=g(x, t) .
$$

Then, we get:

$$
\psi_{n}(x, t)-\psi_{n-1}(x, t)=\frac{\lambda}{\mu} \int_{0}^{t} \int_{\Omega} \Phi(t, \tau) k(|x-y|)\left(\psi_{n-1}(y, \tau)-\psi_{n-2}(y, \tau)\right) \mathrm{d} y \mathrm{~d} \tau .
$$

From Eq. (2), we have:

$$
H_{n}(x, t)=\frac{\lambda}{\mu} \int_{0}^{t} \int_{\Omega} \Phi(t, \tau) k(|x-y|) H_{n-1}(y, \tau) \mathrm{d} y \mathrm{~d} \tau .
$$

Using the properties of the norm, we obtain:

$$
\left\|H_{n}(x, t)\right\| \leq|\gamma|\left\|\int_{0}^{t} \int_{\Omega} \Phi(t, \tau) k(|x-y|) H_{n-1}(y, \tau) \mathrm{d} y \mathrm{~d} \tau\right\| ; \quad \gamma=\frac{\lambda}{\mu} .
$$

For $n=1$, the formula (3) yields:

$$
\left\|H_{1}(x, t)\right\| \leq|\gamma|\left\|\int_{0}^{t} \int_{\Omega} \Phi(t, \tau) k(|x-y|) H_{0}(y, \tau) \mathrm{d} y \mathrm{~d} \tau\right\| .
$$

By applying the Cauchy-Schwarz inequality and using the condition (ii), we get:

$$
\left\|H_{1}(x, t)\right\| \leq|\gamma| M\left\|\left(\int_{\Omega}|k(|x-y|)|^{2} \mathrm{~d} y\right)^{\frac{1}{2}} \cdot \max _{0 \leq t \leq T}\left|\int_{0}^{t}\left(\int_{\Omega}\left|H_{0}(y, \tau)\right|^{2} \mathrm{~d} y\right)^{\frac{1}{2}} \mathrm{~d} \tau\right|\right\| .
$$

Using the conditions (i) and (iii), we have:

$$
\left\|H_{1}(x, t)\right\| \leq|\gamma| M k^{*} N .
$$

By induction, we get:

$$
\left\|H_{n}(x, t)\right\| \leq \beta^{n} N ; \quad \beta=|\gamma| M k^{*}<1 ; \quad n=1,2, \ldots
$$

Since

$$
|\lambda|<\frac{|\mu|}{M k^{*}}
$$

this leads us to say that the sequence $\psi_{n}(x, t)$ has a convergent solution. So that, for $n \rightarrow$ $\infty$, we have:

$$
\psi(x, t)=\sum_{i=0}^{\infty} H_{i}(x, t) .
$$


The above formula represents an infinite convergence series.

Lemma 2 If the conditions (i)-(iii) are satisfied, the function $\psi(x, t)$ of the series (4) represents a unique solution of Eq. (1).

Proof To show that $\psi(x, t)$ is the only solution of Eq. (1), we assume the existence of another solution $\varphi(x, t)$ of Eq. (1), and then, we obtain:

$$
\mu[\psi(x, t)-\varphi(x, t)]=\lambda \int_{0}^{t} \int_{\Omega} \Phi(t, \tau) k(|x-y|)[\psi(y, \tau)-\varphi(y, \tau)] \mathrm{d} y \mathrm{~d} \tau,
$$

which leads us to the following:

$$
\|\psi(x, t)-\varphi(x, t)\|=|\gamma|\left\|\int_{0}^{t} \int_{\Omega} \Phi(t, \tau) k(|x-y|)(\psi(y, \tau)-\varphi(y, \tau)) \mathrm{d} y \mathrm{~d} \tau\right\| .
$$

By applying the Cauchy-Schwarz inequality and using the conditions (i) and (ii), we get:

$$
\begin{aligned}
\|\psi(x, t)-\varphi(x, t)\| & \leq|\gamma| M k^{*} \int_{0}^{t} \int_{\Omega}\|\psi(y, \tau)-\varphi(y, \tau)\| \mathrm{d} y \mathrm{~d} \tau, \\
& \leq \beta\|\psi(x, t)-\varphi(x, t)\| ; \quad \beta=|\gamma| M k^{*}<1 .
\end{aligned}
$$

The formula (5) can be adapted as:

$$
(1-\beta)\|\psi(x, t)-\varphi(x, t)\| \leq 0 .
$$

Since $\beta<1$, so that $\psi(x, t)=\varphi(x, t)$, that is the solution is unique.

\section{Banach's fixed point theorem}

When Picard's method fails to prove the existence of a unique solution for the homogeneous integral equations or for the integral equations of the first kind, we must use Banach's fixed point theorem. For this, we write the formula (1) in the integral operator form:

$$
(\bar{U} \psi)(x, t)=\frac{1}{\mu} g(x, t)+(U \psi)(x, t) ;(U \psi)(x, t)=\frac{\lambda}{\mu} \int_{0}^{t} \int_{\Omega} \Phi(t, \tau) k(|x-y|) \psi(y, \tau) \mathrm{d} y \mathrm{~d} \tau .
$$

To prove the existence of a unique solution of Eq. (1), using Banach's fixed point theorem, we must prove the normality and continuity of the integral operator (6).

(a) For the normality, we use Eq. (6) to get:

$$
\|(U \psi)(x, t)\|=\left|\frac{\lambda}{\mu}\right|\left\|\int_{0}^{t} \int_{\Omega} \Phi(t, \tau) k(|x-y|) \psi(y, \tau) \mathrm{d} y \mathrm{~d} \tau\right\| ; \quad \mu \neq 0 .
$$

Using the condition (ii), then applying the Cauchy-Schwarz inequality, we get:

$$
\|(U \psi)(x, t)\| \leq\left|\frac{\lambda}{\mu}\right| M\left\|\left(\int_{\Omega}|k(|x-y|)|^{2} \mathrm{~d} y\right)^{\frac{1}{2}} \cdot \max _{0 \leq t \leq T}\left|\int_{0}^{t}\left(\int_{\Omega}\left|H_{0}(y, \tau)\right|^{2} \mathrm{~d} y\right)^{\frac{1}{2}} \mathrm{~d} \tau\right|\right\| .
$$

Using the condition (i), we obtain:

$$
\|(U \psi)(x, t)\| \leq\left|\frac{\lambda}{\mu}\right| M k^{*}\|\psi(x, t)\|,
$$


since

$$
\|(U \psi)(x, t)\| \leq \beta\|\psi(x, t)\| ; \quad \beta=\left|\frac{\lambda}{\mu}\right| M k^{*}<1,
$$

where

$$
|\lambda|<\frac{|\mu|}{M k^{*}} .
$$

Therefore, the integral operator $U$ has a normality, which leads immediately after using the condition (iii) to the normality of the operator $\bar{U}$.

(b) For the continuity, we suppose the two potential functions $\psi_{1}(x, t)$ and $\psi_{2}(x, t)$ in the space $L_{2}(\Omega) \times C[0, T]$ are satisfied in Eq. (6), then:

$$
\begin{aligned}
& \left(\bar{U} \psi_{1}\right)(x, t)=\frac{1}{\mu} g(x, t)+\frac{\lambda}{\mu} \int_{0}^{t} \int_{\Omega} \Phi(t, \tau) k(|x-y|) \psi_{1}(y, \tau) \mathrm{d} y \mathrm{~d} \tau, \\
& \left(\bar{U} \psi_{2}\right)(x, t)=\frac{1}{\mu} g(x, t)+\frac{\lambda}{\mu} \int_{0}^{t} \int_{\Omega} \Phi(t, \tau) k(|x-y|) \psi_{2}(y, \tau) \mathrm{d} y \mathrm{~d} \tau .
\end{aligned}
$$

Using Eq. (7), we get:

$$
\bar{U}\left[\psi_{1}(x, t)-\psi_{2}(x, t)\right]=\frac{\lambda}{\mu} \int_{0}^{t} \int_{\Omega} \Phi(t, \tau) k(|x-y|)\left[\psi_{1}(y, \tau)-\psi_{2}(y, \tau)\right] \mathrm{d} y \mathrm{~d} \tau .
$$

Using the condition (ii) and applying the Cauchy-Schwarz inequality, we get:

$$
\begin{aligned}
\left\|\bar{U}\left[\psi_{1}(x, t)-\psi_{2}(x, t)\right]\right\| \leq & \left|\frac{\lambda}{\mu}\right| M \|\left(\int_{\Omega}|k(|x-y|)|^{2} \mathrm{~d} y\right)^{\frac{1}{2}} \\
& \cdot \max _{0 \leq t \leq T}\left|\int_{0}^{t}\left(\int_{\Omega}\left|\psi_{1}(y, \tau)-\psi_{2}(y, \tau)\right|^{2} \mathrm{~d} y\right)^{\frac{1}{2}} \mathrm{~d} \tau\right| \| .
\end{aligned}
$$

By using the condition (i), the last inequality becomes:

$$
\left\|\bar{U}\left[\psi_{1}(x, t)-\psi_{2}(x, t)\right]\right\| \leq\left|\frac{\lambda}{\mu}\right| M k^{*}\left\|\psi_{1}(x, t)-\psi_{2}(x, t)\right\| .
$$

Hence, we have:

$$
\left\|\bar{U}\left[\psi_{1}(x, t)-\psi_{2}(x, t)\right]\right\| \leq \beta\left\|\psi_{1}(x, t)-\psi_{2}(x, t)\right\| ; \quad \beta=\left|\frac{\lambda}{\mu}\right| M k^{*}<1,
$$

with

$$
|\lambda|<\frac{|\mu|}{M k^{*}} .
$$

Inequality (8) leads us to the continuity of the integral operator $\bar{U}$. So that, $\bar{U}$ is a contraction operator. Therefore, by Banach's fixed point theorem, there is an unique fixed point $\psi(x, t)$, which is the solution of the linear mixed integral Eq. (1).

\section{Separation of variables method}

To obtain the general solution of Eq. (1), we do the following:

For $t=0$, the formula (1) becomes:

$$
\mu \psi(x, 0)=g(x, 0) .
$$

Then, seek the solution of Eq. (1) in the form:

$$
\psi(x, t)=\sum_{n=1}^{\infty} c_{n}(t) \psi_{n}(x) .
$$


In this aim, we write:

$$
\psi(x, t)=\psi_{0}(x, t)+\psi_{1}(x, t)
$$

where $\psi_{0}(x, t), \psi_{1}(x, t)$ are called, respectively, the complementary and particular solution of (1). Using Eq. (10) in Eq. (1), we get:

$$
\mu \psi_{k}(x, t)-\lambda \int_{0}^{t} \int_{\Omega} \Phi(t, \tau) k(|x-y|) \psi_{k}(y, \tau) \mathrm{d} y \mathrm{~d} \tau=\delta_{k} g(x, t) ; \quad k=0,1 .
$$

Also, for Eq. (9), we have:

$$
\mu \psi_{k}(x, 0)=\delta_{k} g(x, 0),
$$

where,

$$
\delta_{k}=\left\{\begin{array}{ll}
0 ; & k=0 \\
1 ; & k=1
\end{array} .\right.
$$

From Eqs. (11) and (12), we get:

$$
\mu\left[\psi_{k}(x, t)-\psi_{k}(x, 0)\right]-\lambda \int_{0}^{t} \int_{\Omega} \Phi(t, \tau) k(|x-y|) \psi_{k}(y, \tau) \mathrm{d} y \mathrm{~d} \tau=\delta_{k}[g(x, t)-g(x, 0)] .
$$

Now, we can represent the solution of (10) in the series form:

$$
\psi_{k}(x, t)=\sum_{n=1}^{\infty}\left(c_{2 n}^{(k)}(t) \psi_{2 n}(x)+c_{2 n-1}^{(k)}(t) \psi_{2 n-1}(x)\right)
$$

where $\psi_{2 n}(x), \psi_{2 n-1}(x)$ are the even and odd functions, respectively.

Using Eq. (14) in Eq. (13), we obtain:

$$
\begin{aligned}
& \mu \sum_{n=1}^{\infty}\left(c_{2 n}^{(k)}(t)-c_{2 n}^{(k)}(0)\right) \psi_{2 n}(x)+\mu \sum_{n=1}^{\infty}\left(c_{2 n-1}^{(k)}(t)-c_{2 n-1}^{(k)}(0)\right) \psi_{2 n-1}(x) \\
& -\lambda \int_{0}^{t} \int_{\Omega} \Phi(t, \tau) k(|x-y|) \sum_{n=1}^{\infty}\left(c_{2 n}^{(k)}(\tau) \psi_{2 n}(y)+c_{2 n-1}^{(k)}(\tau) \psi_{2 n-1}(y)\right) \mathrm{d} y \mathrm{~d} \tau \\
& =\delta_{k}[g(x, t)-g(x, 0)] .
\end{aligned}
$$

Taking $k=0$, in Eq. (13), yields:

$$
\begin{aligned}
& \mu \sum_{n=1}^{\infty}\left(c_{2 n}^{(0)}(t)-c_{2 n}^{(0)}(0)\right) \psi_{2 n}(x)+\mu \sum_{n=1}^{\infty}\left(c_{2 n-1}^{(0)}(t)-c_{2 n-1}^{(0)}(0)\right) \psi_{2 n-1}(x) \\
& \quad-\lambda \int_{0}^{t} \int_{\Omega} \Phi(t, \tau) k(|x-y|) \sum_{n=1}^{\infty}\left(c_{2 n}^{(0)}(\tau) \psi_{2 n}(y)+c_{2 n-1}^{(0)}(\tau) \psi_{2 n-1}(y)\right) \mathrm{d} y \mathrm{~d} \tau=0 .
\end{aligned}
$$

Theorem 2 (see [26, 27]). For a symmetric and positive kernel of Fredholm integral term of Eq. (1), the integral operator,

$$
\left(K \psi_{n}\right)(x)=\int_{\Omega} k(|x-y|) \psi_{n}(y) \mathrm{d} y
$$

through the time interval $0 \leq t \leq T<1$ is compact and self-adjoint operator. So, we may write $\left(K \psi_{n}\right)(x)=\alpha_{n} \psi_{n}(x)$, where $\alpha_{n}$ and $\psi_{n}(x)$ are the eigenvalues and the eigenfunctions of the integral operator, respectively. 
In view of Theorem 2 and Eq. (16), we arrive to the following:

$$
\begin{aligned}
& \mu \sum_{n=1}^{\infty}\left(c_{2 n}^{(0)}(t)-c_{2 n}^{(0)}(0)\right) \psi_{2 n}(x)+\mu \sum_{n=1}^{\infty}\left(c_{2 n-1}^{(0)}(t)-c_{2 n-1}^{(0)}(0)\right) \psi_{2 n-1}(x) \\
& -\lambda \int_{0}^{t} \Phi(t, \tau) \sum_{n=1}^{\infty}\left(\alpha_{2 n} c_{2 n}^{(0)}(\tau) \psi_{2 n}(x)+\alpha_{2 n-1} c_{2 n-1}^{(0)}(\tau) \psi_{2 n-1}(x)\right) \mathrm{d} \tau=0 .
\end{aligned}
$$

Separating the odd and even terms, we obtain:

$$
c_{2 n}^{(0)}(t)-\gamma \int_{0}^{t} \alpha_{2 n} \Phi(t, \tau) c_{2 n}^{(0)}(\tau) \mathrm{d} \tau=c_{2 n}^{(0)}(0) ; \quad \gamma=\frac{\lambda}{\mu},
$$

and

$$
c_{2 n-1}^{(0)}(t)-\gamma \int_{0}^{t} \alpha_{2 n-1} \Phi(t, \tau) c_{2 n-1}^{(0)}(\tau) \mathrm{d} \tau=c_{2 n-1}^{(0)}(0) .
$$

Equations (17) and (18) give the same results for even and odd functions, so it is suffice to study the following equation:

$$
c_{n}^{(0)}(t)-\gamma \int_{0}^{t} \alpha_{n} \Phi(t, \tau) c_{n}^{(0)}(\tau) \mathrm{d} \tau=c_{n}^{(0)}(0) ; \quad \gamma=\frac{\lambda}{\mu},
$$

where $c_{n}^{(0)}(0)$ is the constant which will be determined.

Also, taking $k=1$ in formula (15), we obtain:

$$
\begin{aligned}
& \mu \sum_{n=1}^{\infty}\left(c_{2 n}^{(1)}(t)-c_{2 n}^{(1)}(0)\right) \psi_{2 n}(x)+\mu \sum_{n=1}^{\infty}\left(c_{2 n-1}^{(1)}(t)-c_{2 n-1}^{(1)}(0)\right) \psi_{2 n-1}(x) \\
& \quad-\lambda \int_{0}^{t} \int_{\Omega} \Phi(t, \tau) k(|x-y|) \sum_{n=1}^{\infty}\left(c_{2 n}^{(1)}(\tau) \psi_{2 n}(y)+c_{2 n-1}^{(1)}(\tau) \psi_{2 n-1}(y)\right) \mathrm{d} y \mathrm{~d} \tau \\
& \quad=[g(x, t)-g(x, 0)] .
\end{aligned}
$$

Using Theorem 2 in Eq. (20), to have:

$$
\begin{aligned}
& \mu \sum_{n=1}^{\infty}\left(c_{2 n}^{(1)}(t)-c_{2 n}^{(1)}(0)\right) \psi_{2 n}(x)+\mu \sum_{n=1}^{\infty}\left(c_{2 n-1}^{(1)}(t)-c_{2 n-1}^{(1)}(0)\right) \psi_{2 n-1}(x) \\
& -\lambda \int_{0}^{t} \Phi(t, \tau) \sum_{n=1}^{\infty}\left(\alpha_{2 n} c_{2 n}^{(1)}(\tau) \psi_{2 n}(x)+\alpha_{2 n-1} c_{2 n-1}^{(1)}(\tau) \psi_{2 n-1}(x)\right) \mathrm{d} \tau \\
& =\sum_{n=1}^{\infty} a_{2 n} \psi_{2 n}(x)[g(x, t)-g(x, 0)],
\end{aligned}
$$

where,

$$
1=\sum_{n=1}^{\infty} a_{2 n} \psi_{2 n}(x),
$$

the formula (21) can be separated to the following equations:

$$
\begin{gathered}
c_{2 n}^{(1)}(t)-\gamma \int_{0}^{t} \alpha_{2 n} \Phi(t, \tau) c_{2 n}^{(1)}(\tau) \mathrm{d} \tau=\frac{1}{\mu} a_{2 n}[g(x, t)-g(x, 0)]+c_{2 n}^{(1)}(0) ; \quad \gamma=\frac{\lambda}{\mu}, \\
c_{2 n-1}^{(1)}(t)-\gamma \int_{0}^{t} \alpha_{2 n-1} \Phi(t, \tau) c_{2 n-1}^{(1)}(\tau) \mathrm{d} \tau=c_{2 n-1}^{(1)}(0) .
\end{gathered}
$$

Equations (19) and (22) represent the Volterra integral equations of the second kind that have the same continuous kernel $\Phi(t, \tau) \in C([0, T] \times[0, T])$, and each of them has a 
unique solution in the class $C[0, T]$; the books edited by Linz [28] and Burton [29] contain many different methods to solve the integral Eqs. (19) and (22).

The values of $c_{n}^{(0)}(0), c_{2 n}^{(1)}(0)$, and $c_{2 n-1}^{(1)}(0)$ can be obtained, we return to Eq. (9), and we seek the solution of this equation in the form:

$$
\psi(x, 0)=\sum_{n=1}^{\infty} c_{n}(0) \psi_{n}(x)
$$

Hence, in this respect, we write:

$$
\psi(x, t)=\psi_{0}(x, t)+\psi_{1}(x, t),
$$

where $\psi_{0}(x, t)$ is a complementary solution while $\psi_{1}(x, t)$ is a particular solution. So, from Eq. (9), we write:

$$
\mu \psi_{k}(x, 0)=\delta_{k} g(x, 0),
$$

and expand the solution of Eq. (23) in the form:

$$
\psi_{k}(x, 0)=\sum_{n=1}^{\infty}\left(c_{2 n}^{(k)}(0) \psi_{2 n}(x)+c_{2 n-1}^{(k)}(0) \psi_{2 n-1}(x)\right) .
$$

Using Eq. (25) in Eq. (24), we obtain:

$$
\mu \sum_{n=1}^{\infty}\left(c_{2 n}^{(k)}(0) \psi_{2 n}(x)+c_{2 n-1}^{(k)}(0) \psi_{2 n-1}(x)\right)=\delta_{k} g(x, 0) .
$$

If we take $k=0$ in Eq. (26), we obtain:

$$
\mu \sum_{n=1}^{\infty}\left(c_{2 n}^{(0)}(0) \psi_{2 n}(x)+c_{2 n-1}^{(0)}(0) \psi_{2 n-1}(x)\right)=0 .
$$

Equating the odd and even terms in both sides, we get:

$$
c_{2 n}^{(0)}(0)=0, \quad c_{2 n-1}^{(0)}(0)=0 .
$$

Then, we have:

$$
c_{n}^{(0)}(0)=0 .
$$

Taking $k=1$ in Eq. (26), we have:

$$
\mu \sum_{n=1}^{\infty}\left(c_{2 n}^{(1)}(0) \psi_{2 n}(x)+c_{2 n-1}^{(1)}(0) \psi_{2 n-1}(x)\right)=g(x, 0) .
$$

Equating both sides of the last equation, we get:

$$
c_{2 n}^{(1)}(0)=0, \quad c_{2 n-1}^{(1)}(0)=0,
$$

so the last two formulas give us:

$$
c_{n}^{(1)}(0)=0 .
$$

In view of Eqs. (19) and (22), the general solution of (1) can be adapted in the form:

$$
\psi_{N}(x, t)=\sum_{n=1}^{N}\left(c_{n}^{(0)}(t)+c_{n}^{(1)}(t)\right) \psi_{n}(x),
$$

where $c_{n}^{(0)}(t)$ and $c_{n}^{(1)}(t)$ must satisfy the inequality:

$$
\sum_{n=1}^{N}\left|c_{n}^{(0)}(t)+c_{n}^{(1)}(t)\right|<\epsilon, \quad(N \rightarrow \infty, \epsilon \ll 1,0 \leq t \leq T<1) .
$$


Theorem 3 If, for $t \in[0, T]$, the inequality (28) holds, the series (27) is uniformly convergent in the space $\ell_{2}(\Omega) \times C[0, T], N \rightarrow \infty$. Hence, the solution of the Volterra-Fredholm integral Eq. (1) can be obtained in a series form of (27).

Theorem 4 For the given functions $g(x, t) \in L_{2}(\Omega) \times C[0, T], \Phi(t, \tau) \in$ $C([0, T] \times[0, T]), k(x, y) \in C([\Omega] \times[\Omega])$, and under the condition (28), we have:

$$
\left\|\psi(x, t)-\psi_{N}(x, t)\right\| \rightarrow 0 \text { as } N \rightarrow \infty,
$$

where $\psi(x, t)$ represents the unique solution of Eq. (1) and the error takes the form:

$$
E_{N}=\left\|\psi(x, t)-\psi_{N}(x, t)\right\|
$$

where

$$
E_{N} \rightarrow 0 \text { as } N \rightarrow 1
$$

\section{Conclusion and remarks}

From the above results and discussion, the following may be concluded:

1 Equation (1) has a unique solution $\psi(x, t)$ in the space $L_{2}(\Omega) \times C[0, T]$, under some conditions.

2 The existence of a unique solution of the Volterra-Fredholm integral equation is discussed and proved using Picard's method and Banach's fixed point method.

3 The Volterra-Fredholm integral equation of the second kind, in time and position, after using separation of variables method leads to the Volterra integral equations of the second kind with a continuous kernel.

4 Solutions of the Volterra integral equations can be obtained by numerical methods.

Acknowledgements

We would like to thank Dr. M. A. Nasr, (Dept. of Maths. College of Science and Arts-Gurayat, Jouf University, KSA) and the anonymous reviewers for their constructive suggestions towards upgrading the quality of the manuscript.

\section{Authors' contributions}

All parts contained in the research were carried out by the authors through hard work and a review of the various

references and contributions in the field of mathematics and applied physics. All authors are read and approved the final manuscript.

Funding

No funding was received.

Availability of data and materials

Not applicable.

Ethics approval and consent to participate

Not applicable.

\section{Consent for publication}

Not applicable.

Competing interests

The authors declare that they have no competing interests.

\section{Author details}

${ }^{1}$ Department of Mathematics, Faculty of Education, Alexandria University, Alexandria, 21511, Egypt. ${ }^{2}$ Department of Mathematics, Faculty of Science, Benha University, Benha, 13518, Egypt.

Received: 24 July 2019 Accepted: 17 February 2020

Published online: 28 February 2020 


\section{References}

1. Bazm, S.: Bernoulli polynomials for the numerical solution of some classes of linear and nonlinear integral equations. J. Comput. Appl. Math. 275, 44-60 (2015)

2. Brauer, F., Castillo-Chvez, C.: Mathematical Models in Population Biology and Epidemiology. Springer, New York (2012)

3. Craciun, C., Serban, M. A.: A nonlinear integral equation via picard operators. Fixed Point Theory. 12(1), 57-70 (2011)

4. Hamoud, A. A., Ghadle, K. P.: Approximate solutions of fourth-order fractional integro-differential equations. Acta Univ. Apulensis. 55, 49-61 (2018)

5. Hamoud, A. A., Ghadle, K. P.: On the numerical solution of nonlinear Volterra-Fredholm integral equations by variational iteration method. Int. J. Adv. Sci. Tech. Res. 3, 45-51 (2016)

6. Ilea, V., Otrocol, D.: Some properties of solutions of a functional-differential equation of second order with delay. Sci. World J. 2014 (2014). https://doi.org/10.1155/2014/878395

7. Otrocol, D., llea, V.: Ulam stability for a delay differential equation. Cent. Eur. J. Math. 11(7), 1296-1303 (2013)

8. Rus, I. A.: Results and problems in Ulam stability of operatorial equations and inclusions. In: Handbook of Functional Equations, pp. 323-352. Springer Optim. Appl, New York, (2014)

9. Abdou, M. A., Nasr, M. E., Abdel-Aty, M. A.: A study of normality and continuity for mixed integral equations. J. Fixed Point Theory Appl. 20(1), 5 (2018). https://doi.org/10.1007/s11784-018-0490-0

10. Abdou, M. A., Nasr, M. E., Abdel-Aty, M. A.: Study of the normality and continuity for the mixed integral equations with phase-lag term. Int. J. Math. Anal. 11(16), 787-799 (2017). https://doi.org/10.12988/ijma.2017.7798

11. Karapinar, E., Kumari, P., Lateef, D.: A new approach to the solution of the Fredholm integral equation via a fixed point on extended b-metric spaces. Symmetry. 10(10), 512 (2018)

12. Hammad, H. A., De la Sen, M.: A solution of fredholm integral equation by using the cyclic $\eta_{q}^{s}$-rational contractive mappings technique in b-metric-like spaces. Symmetry. 11(9), 1184 (2019)

13. Tunç, C., Tunç, O.: On behaviours of functional volterra integro-differential equations with multiple time lags. J. Taibah Univ. Sci. 12(2), 173-179 (2018)

14. Tunç, C., Tunç, O.: A note on the qualitative analysis of volterra integro-differential equations. J. Taibah Univ. Sci. 13(1), 490-496 (2019)

15. Basseem, M.: Degenerate method in mixed nonlinear three dimensions integral equation. Alex. Eng. J. 58(1), 387-392 (2019)

16. AL-Jawary, M., Radhi, G., Ravnik, J.: Two efficient methods for solving Schlömilch's integral equation. Int. J. Intell. Comput. Cybernet. 10(3), 287-309 (2017)

17. Cattani, C., Kudreyko, A.: Harmonic wavelet method towards solution of the Fredholm type integral equations of the second kind. Appl. Math. Comput. 215(12), 4164-4171 (2010)

18. Gu, Z., Guo, X., Sun, D.: Series expansion method for weakly singular Volterra integral equations. Appl. Numer. Math. $105,112-123(2016)$

19. Hashemizadeh, E., Rostami, M.: Numerical solution of Hammerstein integral equations of mixed type using the Sinc-collocation method. J. Comput. Appl. Math. 279, 31-39 (2015)

20. Maleknejad, K., Almasieh, H., Roodaki, M.: Triangular functions (TF) method for the solution of nonlinear Volterra-Fredholm integral equations. Commun. Nonlinear Sci. Numer. Simul. 15(11), 3293-3298 (2010)

21. Nasr, M. E., Abdel-Aty, M. A.: Analytical discussion for the mixed integral equations. J. Fixed Point Theory Appl. 20(3), 115 (2018). https://doi.org/10.1007/s11784-018-0589-3

22. Sizikov, V., Sidorov, D.: Generalized quadrature for solving singular integral equations of abel type in application to infrared tomography. Appl. Numer. Math. 106, 69-78 (2016)

23. Abdou, M. A., Al-Kader, G. M.: Mixed type of integral equation with potential kernel. Turk. J. Math. 32(1), 83-101 (2008)

24. Haagerup, U.: Lp - spaces associated with an arbitrary von neumann algebra. In: Algebres D’opérateurs et leurs applications en physique mathématique (Colloques internationaux C.N.R. S., Paris, 1948), vol. 274, pp. 175-184, (1979)

25. Muscat, J.: Functional Analysis: an Introduction to Metric Spaces, Hilbert Spaces, and Banach Algebras. Springer, New York Dordrecht London (2014)

26. Abdou, M. A.: Fredholm-Volterra integral equation of the first kind and contact problem. Appl. Math. Comput. 125(2-3), 177-193 (2002)

27. Popov, G. Y.: Contact Problems for a Linearly Deformable Base. Odessa, Kiev (1982)

28. Linz, P.: Analytical and Numerical Methods for Volterra Equations, vol. 7. Society for Industrial and Applied Mathematics, Siam (1985)

29. Burton, T. A.: Volterra Integral and Differential Equations, vol. 202. Elsevier, USA (2005)

\section{Publisher's Note}

Springer Nature remains neutral with regard to jurisdictional claims in published maps and institutional affiliations. 